\title{
Evaluación de la dificultad percibida de realizar conductas proambientales
}

\section{Valuation of perceived difficulty to performance pro- environmental behaviours}

\author{
María C. Vanegas Rico ${ }^{1}$ \\ ${ }^{1}$ Facultad de Estudios Superiores Zaragoza, UNAM, CDMX, México \\ Correo electronico:ma.cristina.vanegas@gmail.com
}

\begin{abstract}
Resumen
Realizar conductas proambientales (CPA) conlleva un costo conductual, el cual se puede plantear en términos de la dificultad que tiene para la persona llevar a cabo la acción. El objetivo del estudio fue evaluar la dificultad percibida de diferentes acciones proambientales cotidianas a partir de dos fuentes: la jerarquización de conductas y el reporte de su ejecución. Se repartieron treinta conductas proambientales en dos formatos, cada uno se aplicó a una muestra diferente $\left(\mathrm{N}_{1}=168\right.$ y $\left.\mathrm{N}_{2}=168\right)$, todos habitantes de la Zona Metropolitana del Valle de México, con edad superior a 18 años. La jerarquización se analizó con el método de porcentaje relativo de prioridad, y la ejecución de las conductas con el modelo Rasch para estimar el nivel de dificultad. Los resultados mostraron que las conductas que se percibieron más difíciles y presentaron un índice de dificultad alto, se vinculan a la movilidad y las compras verdes; mientras que las más fáciles fueron acciones altamente promocionadas en México: no tirar basura en la calle, separar los residuos o apagar la luz que no se utiliza. Se reflexionan las implicaciones de los métodos de análisis utilizados, así como la aportación al conocimiento del costo conductual de la CPA.
\end{abstract}

Palabras clave: Conducta proambiental, costo conductual, jerarquización, modelo Rasch.

\begin{abstract}
Performing pro-environmental behaviors (PEB) has a behavioral cost, which can be propose as the difficulty that the action hold for people. The aim of this study was to evaluate perceived difficulty of various daily pro-environmental behaviors from two sources: hierarchy of behaviors and their execution report. Thirty pro-environmental behaviors were distributed in two formats, each of them was applied to a different sample (the first $\mathrm{N}_{1}=168$ and the second $\mathrm{N}_{2}=$ 168), all of them are habitants of the Mexico Valley Metropolitan Area, with an age superior to 18 years old. Hierarchy was analyzed with the relative priority percentage method, and the performing of behaviors was analyzed
\end{abstract}


with the Rasch model to estimate the difficulty level. The results showed that the most difficult behaviors perceived and presented a high difficulty index, are linked to mobility and green shopping, meanwhile, the easiest behaviors were actions highly promoted in Mexico: not littering, waste separation behavior, or turn off lights. The implications of analysis methods used are discussed, as well as the contributions to the knowledge of the behavioral cost of PEB.

Keywords: Pro-environmental behaviour, behavioral cost, hierarchy, Rasch' model.

\section{Introducción}

En el estudio de la conducta proambiental (CPA), una premisa es que si las personas presentan factores personales que las dispongan favorablemente a la acción proambiental, entonces será más probable que la lleven a cabo (Bamberg \& Möser, 2007; Corral, 2001; Gifford, 2014; Steg \& Vlek, 2009). Sin embargo, también se ha aceptado que aparece una brecha entre la disposición y la acción (Kollmuss \& Agyeman, 2002), por la cual las personas no son tan proambientales como sus actitudes.

Diekmann y Preisendörfer (1998, 2003) propusieron la hipótesis de bajo costo, según la cual la relación entre actitudes y conducta puede ser afectada por el costo de realizar esa conducta, que incluye aspectos como el esfuerzo, la conveniencia, y/o la dificultad (o facilidad) percibida. El efecto es de un moderador por el cual, si el costo es alto, se apreciará una débil relación actitud-conducta, mientras que, si el costo es bajo, la relación será fuerte. De forma semejante, Bagozzi et al. (1990) sugirieron que el nivel de esfuerzo funciona como moderador entre actitud y conducta, definiendo al esfuerzo como el grado de dificultad por ejecutar una conducta. Extrapolando la idea, mientras una acción es más difícil de realizar se considerará más costosa.

Si bien otros investigadores no obtuvieron resultados que apoyen tal cual la hipótesis de bajo costo (Best \& Kneip, 2011; Kaiser \& Schultz, 2009), las evidencias y reflexiones de quienes investigan CPA parecen concordar con la existencia de un efecto del costo en la ejecución de la conducta (Anderson \& von Borstede, 2010; Steg \& Vlek, 2009). Además, se ha encontrado que percibir que una CPA es fácil de llevar a cabo favorece la posibilidad de que se realice (Fujii, 2006; Fujii \& Taniguchi, 2013). De aquí se deriva el interés por conocer cuáles CPA's se perciben fáciles y/o cuáles difíciles.

Al respecto, Fujii (2006) empleó la variable facilidad percibida de implementación, midiéndola con un solo reactivo para cada una de cuatro acciones (reducción de electricidad, gas, basura y automóvil); el autor encontró que la facilidad percibida tenía el mayor poder predictivo en cada conducta, y que la reducción en el uso de automóvil es una de las conductas más difíciles. Acorde con esto, Diekmann y Preisendörfer (1998) consideraron que la separación de residuos y las compras verdes son de bajo costo, en tanto que las conductas de movilidad y energía son de alto costo, para ello tomaron como referencia la frecuencia con que se reportan las acciones, deduciendo que aquellas que son esporádicas o inusuales son más costosas (Diekmann \& Preisendörfer, 2003). Tomar la frecuencia de reporte como indicador de su dificultad (o costo) tiene lógica, y se ha 
utilizado por otros autores (Kaiser \& Schultz, 2009), sin embargo, puede no ser suficiente.

Kaiser, retomando el Paradigma de Campbell, propuso que la actitud puede corresponder con la expresión de acciones proambientales, a partir de tener en cuenta el nivel de dificultad de la conducta, el cual se podría estimar empleando el modelo de medición Rasch, considerado parte de los modelos de la teoría de respuesta al ítem (Kaiser et al., 2010). Este método permite conocer la probabilidad de elegir una respuesta a partir del nivel de habilidad de la persona y la dificultad del reactivo, que trasladado a la CPA se referiría al grado en el que el individuo está inclinado a favor del cuidado del ambiente y la dificultad de las acciones proambientales (Byrka, et al, 2017; Kaiser \& Byrka, 2015).

Buscando ampliar el conocimiento sobre el costo conductual de la CPA a partir de la dificultad percibida, este estudio tuvo el objetivo de evaluar la dificultad percibida de diferentes acciones proambientales cotidianas a partir de dos fuentes: la jerarquización de conductas y el reporte de su ejecución.

\section{Método}

\section{Participantes}

Se llevó a cabo un muestreo no probabilístico por conveniencia, el único criterio de inclusión fue tener una edad igual o mayor a 18 años, bajo la perspectiva de que, al menos legalmente, poseen más independencia para tomar decisiones de ejecutar CPA. Se obtuvo una muestra de 336 habitantes de la Zona Metropolitana del Valle de México (Ciudad de México y zona conurbada del Estado de México), la mitad de ellos recibió el formato 1 y la otra mitad el formato 2. Los respondientes del formato 1 tenían una edad promedio de 35.13 años $(\mathrm{DE}=3.73), 58.9 \%$ fueron mujeres y $38.7 \%$ hombres $(2.4 \%$ de omisiones); la distribución de la escolaridad fue: primaria $5.4 \%$, secundaria $12.5 \%$; medio superior $44.6 \%$, superior $34.5 \%$, y posgrado $1.8 \%$. Los participantes del formato 2 presentaron una edad promedio de 34.96 años ( $\mathrm{DE}=3.87$ ), el $54.2 \%$ fueron mujeres y $42.9 \%$ hombres ( $3 \%$ de omisión); la escolaridad se distribuyó: primaria 4.8\%, secundaria $16.1 \%$; medio superior $36.9 \%$, superior $35.7 \%$, y posgrado $3.6 \%$.

\section{Instrumentos}

Para este estudio se generaron, ex profeso, dos listas de 15 conductas proambientales cada una, que se consideran cotidianas para las personas en entornos de ciudad, recopilando estas acciones de diferentes estudios de CPA (Bamberg \& Möser, 2007; Corral, 2001; Gifford, 2014). Para la evaluación de la jerarquía de las conductas por su dificultad se les dio la instrucción de que ordenaran las 15 acciones de acuerdo con la facilidad que les supone llevarlas a cabo cotidianamente, asignando un 1 a la más fácil y así hasta el valor 15 para la más difícil; para la medición de la realización de las conductas se pidió que indicaran con una marca de verificación (poner una paloma) aquellas acciones que llevan a cabo con una frecuencia de tres o más veces a la semana. Cada forma de respuesta se colocaba en una columna que se encontraba a la derecha de las conductas. Estas listas y sus instrucciones conformaron el instrumento de medición para los propósitos del estudio y se identificaron como formato 1 y 2 (en la discusión se explica la razón de presentar dos formatos). El formato 1 se compone de acciones de transporte, ahorro de agua y luz, así como limpieza del entorno; mientras que el formato 2 se compone de acciones 
sobre compras verdes (productos de bajo impacto ambiental), forma de consumo, y forma de desecho de los residuos, en los resultados se presentan todas las conductas. Aunque las conductas provienen de escalas con propiedades psicométricas de confiabilidad y validez de constructo, no fue el objetivo del presente estudio confirmar o generar nuevas escalas de conducta proambiental, por lo cual no se desarrollaron análisis estadísticos correspondientes para las listas de conductas.

En cada formato se incluyeron preguntas sobre edad, sexo y escolaridad para la descripción de la muestra.

\section{Procedimiento}

En los meses de mayo a junio de 2019 se acudió a lugares públicos de la Ciudad de México (parques y jardines) donde se les pidió a las personas que participaran en el estudio, verificando que se trataran de personas con 18 años o más. A quienes aceptaban responder se les entregaba un formato impreso junto con un lápiz y una tabla sujeta-papel para apoyarse, no se entregó hoja de consentimiento informado, la petición e información fue únicamente verbal. De forma aleatoria se asignó el formato 1 o 2 (solo uno por participante). El tiempo de respuesta fue de 7 a 10 minutos aproximadamente.

\section{Análisis estadístico}

Para observar si hay diferencias entre las muestras del formato 1 y 2 se realizó una prueba t de Student para la edad, y pruebas $X^{2}$ para la distribución de sexo y escolaridad. Para la jerarquización se utilizó el análisis de porcentaje relativo de prioridad (PRP) propuesto por Martínez-Guerrero y Sánchez-Sosa (1981), el cual consiste en asignar un peso de acuerdo con la posición del reactivo (en este caso, la posición 15 tiene el peso más alto para representar la acción más difícil), éste se multiplica por la frecuencia con que se le asignó esa posición; después se suman todos los productos y se dividen entre un valor obtenido al multiplicar la cantidad total de participantes por el peso máximo posible para cualquier reactivo; el cociente obtenido se multiplica por 100 para obtener el PRP. Se empleó el programa Excel de la paquetería Office 360.

Para el análisis de la ejecución de conductas se utilizó el análisis Rasch empleando el programa Ministep versión 4.8.1.0, el cual utiliza la estimación de máxima verosimilitud conjunta. Se trata de una versión gratuita de Winistep, con la limitante de 75 casos, por lo cual se eligieron al azar 75 participantes por cada formato. Se reporta el parámetro de dificultad del ítem $(\delta)$.

No se realizó ningún análisis para establecer confiabilidad y validez de las listas, aunque se reporta la confiabilidad de los ítems que genera el análisis Rasch.

\section{Resultados}


Las características descriptivas de los participantes señalan que las muestras de cada formato son similares al no presentar diferencias significativas: estuvieron conformadas por personas jóvenes adultas en un rango de 30 a 40 años $\left(\mathrm{t}_{(333)}=0.402\right.$; $\mathrm{p}=.688)$, con una proporción ligeramente mayor de mujeres $\left(X_{(1)}^{2}=0.691 ; \mathrm{p}=.406\right)$, y una escolaridad principalmente de medio superior y superior $\left(X_{(4)}^{2}=3.049 ; \mathrm{p}=.550\right)$.

El análisis de PRP permitió ver de forma simple cuál es, en este caso, la CPA que en mayor porcentaje se considera más difícil de entre las opciones presentadas. En la tabla 1 se observa el resultado para el formato 1, las acciones fueron ordenadas de mayor a menor porcentaje resultante, y la letra a la izquierda de la CPA permite conocer cuál fue su posición en el formato de respuesta. Se puede observar que las tres conductas consideradas más difíciles fueron el uso de bicicleta (tanto en trayectos cortos, como uso principal) y el cuidado de áreas verdes cercanas, mientras que las tres conductas más fáciles fueron el cuidado del agua con duchas cortas, cuidado de la luz al apagar la que no se utiliza, y nuevamente el cuidado del agua con el uso de un vaso para el lavado de dientes.

En el caso del formato 2 las tres conductas jerarquizadas con más dificultad se refirieron a la compra de productos verdes: la ubicación de tiendas especializadas o de dichos productos en tiendas comunes, y la compra de aquellos que son biodegradables. Mientras que las acciones con mayor facilidad fueron relacionadas a los residuos: evitar tirarlos en la calle, separarlos en dos tipos y la reutilización de envases (ver tabla 2).

Por su parte, el análisis en modelo Rasch presentó una confiabilidad entre reactivos de 0.94 para el formato 1 . En la tabla 1 se han agregado los valores del nivel de dificultad en escala logit que va -en general- de $-3 \mathrm{a}+3$, donde un valor negativo indica reactivos más fáciles, y valores positivos señalan reactivos más difíciles. Como puede observarse, usar la bicicleta como principal medio de transporte tiene el valor más alto y cercano a +3 , por lo cual se considera una conducta muy difícil, mientras que una conducta demasiado fácil es apagar las luces que no se utilizan. Se han sombreado las casillas que coinciden en el orden de PRP y el índice de dificultad. En general, se aprecian pocas conductas con un índice de dificultad alto (mayor a 2), la mayoría poseen índices moderados en ambos sentidos (facilidad y dificultad). Aunque solo el $26.66 \%$ de las conductas concuerdan en la percepción de la dificultad y el nivel de dificultad obtenido por modelo Rasch, podría considerarse que las restantes son cercanas en posición, la mayor disimilitud estaría en la conducta de limpieza de la calle donde se habita, la cual se jerarquiza en el cuarto lugar de dificultad, pero tiene un valor logit negativo de -0.06 , es decir, ni fácil ni difícil, solo una leve tendencia a ser fácil.

Para el formato 2, el análisis presentó una confiabilidad entre reactivos de 0.96. En la tabla 2 se observa que la conducta con mayor índice de dificultad fue la ubicación de tiendas especializadas en productos ecológicos, que coincide en el primer lugar del PRP, su valor de 3.16 queda por encima de los valores comunes de la escala, lo cual indica que se trata de una acción realmente difícil; algo semejante -pero en sentido contrario- ocurre con la conducta con el menor índice de dificultad, que es evitar tirar basura en la calle, su valor de -3.68 indica una conducta demasiado fácil, y coincide en la jerarquización, como la más fácil de las 15 acciones. De hecho, en este formato casi la mitad de las acciones (46.66\%) coinciden en la posición por el PRP y el nivel de dificultad, además de que se 
observa que la primera mitad de la lista tiene valores logit positivos y la segunda mitad negativos, es decir, que van de difíciles a fáciles, conforme también se van jerarquizando más fáciles.

\section{Tabla 1}

Formato 1. Porcentaje relativo de prioridad e índice de dificultad

\begin{tabular}{|c|c|c|}
\hline Conductas proambientales & PRP & $\delta$ \\
\hline h. Usar bicicleta como principal medio de transporte. & 83.214 & 2.69 \\
\hline g. Usar bicicleta como medio de transporte sólo en trayectos cortos. & 71.190 & 2.12 \\
\hline n. Cuidar de áreas verdes cercanas a casa. & 66.587 & 1.13 \\
\hline o. Barrer y recoger basura de la calle donde habita. & 63.571 & -0.06 \\
\hline e. Lavar los trastes con el equivalente a una cubeta de agua o menos. & 58.016 & 0.81 \\
\hline d. Revisar las instalaciones en caso de posibles fugas. & 57.817 & 0.73 \\
\hline j. Evitar el uso del microondas. & 55.794 & -0.72 \\
\hline k. Desconectar la mayor cantidad de aparatos eléctricos por la noche. & 54.286 & -0.06 \\
\hline i. Desconectar los aparatos eléctricos que no estén en uso. & 49.246 & -0.50 \\
\hline b. Reutilizar el agua del lavado de ropa. & 48.135 & 0.44 \\
\hline c. Recaudar el agua de la regadera hasta que sale caliente. & 45.992 & 0.01 \\
\hline m. Tener focos ahorradores. & 41.389 & -1.50 \\
\hline f. Utilizar un vaso de agua para lavarse los dientes. & 38.413 & -1.40 \\
\hline 1. Apagar la luz que no se utiliza. & 37.460 & -2.07 \\
\hline a. Bañarse en menos de 10 minutos. & 28.849 & -1.60 \\
\hline
\end{tabular}


Tabla 2

Formato 2. Porcentaje relativo de prioridad e índice de dificultad

\begin{tabular}{|c|c|c|}
\hline Conductas proambientales & PRP & $\delta$ \\
\hline a. Ubicar tiendas especializadas en productos ecológicos. & 83.135 & 3.16 \\
\hline c. Ubicar en las tiendas comunes productos ecológicos. & 75.794 & 2.34 \\
\hline b. Comprar productos que indican ser biodegradables. & 68.413 & 0.91 \\
\hline i. Aprovechar los residuos de alimentos en una composta. & 64.127 & 1.10 \\
\hline d. Comprar únicamente detergentes que no dañen el ambiente. & 63.214 & 1.31 \\
\hline e. Comprar desechables de cartón en vez de plástico o unicel. & 61.071 & 1.10 \\
\hline h. Evitar consumir en lugares que usan platos y/o vasos de unicel. & 60.675 & 0.82 \\
\hline $\begin{array}{l}\text { m. Llevar los residuos separados a lugares de recolección } \\
\text { específicos. }\end{array}$ & 58.532 & 0.82 \\
\hline f. Planificar lo que se va a comprar y no adquirir nada más. & 46.706 & -0.91 \\
\hline j. No tirar el aceite de cocina directamente en el drenaje. & 42.579 & -1.41 \\
\hline g. Llevar bolsa para las compras (de tela, lona u otro material). & 42.262 & -1.26 \\
\hline $\begin{array}{l}\text { k. Utilizar un envase (cilindro, cantimplora, etc) para tomar agua } \\
\text { en vez de comprar botellas desechables. }\end{array}$ & 38.690 & -1.63 \\
\hline n. Reutilizar frascos y envases. & 37.302 & -1.33 \\
\hline 1. Separar la basura en orgánica e inorgánica. & 34.048 & -1.33 \\
\hline o. Evitar tirar basura en la calle. & 23.016 & -3.68 \\
\hline
\end{tabular}

\section{Discusión}

Desde la lógica más simple, mientras más difícil es una actividad, se realizará con menor frecuencia o menos personas la llevarán a cabo, y la CPA no es la excepción, por ejemplo, se ha reflexionado que las conductas proambientales de mayor impacto por lo general son las de mayor costo (Fujii \& Taniguchi, 2013), como es la reducción del uso de transporte motorizado particular, cuyas intervenciones han tenido poco éxito general (Fujii, 2006; Fujii \& Taniguchi, 2013).

El interés de este estudio se centró en indagar en el costo conductual a partir de su dificultad percibida, ya que el costo de la CPA es más complejo de lo que podría parecer; por ejemplo, para Diekmann y Preisendörfer (2003) el reciclaje es de bajo costo, pero para Anderson y von Borstede (2010) reciclar supone diversas conductas que pueden percibirse de mayor o menor costo. 
Para responder al objetivo de este estudio se eligió, por una parte, un método de jerarquización para reducir o evitar un posible sesgo positivo debido a que la CPA es socialmente bien vista e inclina a valoraciones positiva, como sería considerarlas más fáciles de lo que realmente suponen para las personas cuando ya deben de ejecutarse, mientras que la respuesta de jerarquización exige que se acepte un mayor nivel de dificultad para algunas conductas en relación con otras. El método de análisis de PRP (MartínezGuerrero y Sánchez-Sosa, 1981), si bien no ha sido aplicado de forma extensa, responde adecuadamente a los intereses de la investigación, con el beneficio de la sencillez de su procedimiento, e incluso permite analizar la varianza de la respuesta entre grupos, lo cual es útil para -a futuro- considerar el estudio por grupos de edades, escolaridad o nivel socioeconómico.

El otro método elegido posee un mayor trasfondo empírico y teórico (Kaiser \& Byrka, 2015; Kaiser et al., 2010), retomando la propuesta de Kaiser, con la cual se puede obtener un índice de dificultad para las conductas proambientales que permite ir estableciendo una valuación cuantitativa del costo conductual, y por la naturaleza de la teoría IRT estos parámetros son independientes a los respondientes.

Respecto a lo encontrado, la ubicación de tiendas especializadas en productos ecológicos se destacó por su alto nivel de dificultad, y se junta con otras conductas de consumo verde que fueron consideradas difíciles, mientras que para Diekmann y Preisendörfer (2003) las compras son de bajo costo, lo cual puede reflejar una diferencia en el apoyo que dan los países a las opciones de productos proambientales, ya que en México parece ya existir una disposición hacia la compras verdes, de acuerdo con un estudio de mercado que indica que el $71 \%$ de los mexicanos busca productos y empaques que sean reciclados, reutilizables o mínimos (Venegas, 2019), además, puede notarse que la acción de planificar las compras se consideró ligeramente fácil. Pero la existencia de tiendas especializadas en México parece estrecharse a lugares que se establecen en áreas de alto nivel socioeconómico, y muchos productos que se publicitan como alternativas verdes comúnmente tienen un precio más alto que las opciones tradicionales.

Por su parte, el uso de bicicleta como medio de transporte resultó calificada como una conducta difícil por ambos métodos y se corrobora la idea de que utilizar medios alternativos al automóvil son costosos (Diekmann \& Preisendörfer, 2003; Fujii \& Taniguchi, 2013), particularmente en grandes metrópolis como la CDMX, que a pesar de que actualmente tiene avances en la implementación de ciclovías (Secretaría de Movilidad [SEMOVI], s.f.), sigue siendo una urbe que da prioridad a los vehículos automotores, y tiene una cultura ciclista aún incipiente.

En el otro extremo, algunas de las conductas que fueron calificadas como más fáciles han sido de las que más se han promovido en el país y puntualmente en la CDMX. Por ejemplo, desde la década de los 70's del siglo pasado se generaron campañas para no tirar basura en la calle, enfatizando cada año en época de lluvias la importancia de las calles limpias para evitar la obstrucción del drenaje y las inundaciones en calles y casas. Otro caso es la separación de residuos en orgánica e inorgánica, donde la CDMX ha implementado como obligatoria la separación, primero en estas dos categorías, y actualmente en cuatro (Norma nadf-024-ambt-2013, 2015). Así como la disposición gubernamental a cambiar los focos incandescentes por ahorradores (Comisión Nacional para el Uso Eficiente de la Energía, 2017). 
Las conductas con nivel de dificultad intermedio son variadas en cuanto al recurso que se quiere conservar o la acción básica a realizar. Algunas conductas se refieren a evitar usar o hacer algo (e.g. usar microondas), otras a la supervisión para evitar un gasto innecesario del recurso (e.g. apagar aparatos eléctricos, revisar fugas). Esto puede ayudar a explicar por qué en los estudios donde se trata de un mismo recurso las escalas no se integran unifactorialmente, o que en los instrumentos de conductas variadas no siempre parecen tener coherencia los factores que se conforman durante el análisis estructural; por ejemplo, en la conservación de la energía se hace la distinción entre acciones de reducción y de eficiencia, pero Starke et al. (2020) consideraron retomar también el paradigma de Campbell y analizar acciones de ahorro de energía con el modelo Rasch para optimizar la evaluación. Esto lleva a otra reflexión en cuanto al uso del modelo Rasch: una de sus premisas es que los ítems deben conformarse unifactorialmente, de hecho, Kaiser propone que las conductas proambientales deben ser vistas así, y lo que las distingue es su nivel de dificultad, es decir, caen en distintos niveles, pero responden a la misma dimensión (Kaiser et al., 2010), mientras que la postura de la presente investigación es que el costo de las conductas puede ayudar a generar categorías conductuales para la CPA.

Finalmente, se reconoce la existencia de varias limitaciones claras: la representatividad de la muestra (en cuanto a tamaño y forma de muestreo); la aplicación sin consentimiento informado; la cantidad de conductas evaluadas y el criterio para elegir éstas y no otras acciones; mayor profundidad y aprovechamiento del modelo Rasch; así como la contrastación de los resultados con el reporte de CPA de otros estudios. Al respecto de los formatos de 15 acciones cada una, se decidió esta presentación debido a que, mientras más ítems se deben jerarquizar, se vuelve más complejo elegir la posición en la parte intermedia al tener que ponderar mentalmente muchas opciones, por ello no sería conveniente solicitar el ordenamiento de 30,50 o más acciones, pero es preciso aplicar mayor sistematicidad en la elección de las conductas que serán incluidos en próximos estudios.

El presente estudio se considera solo un paso en la comprensión del costo de la CPA, se espera en futuras investigaciones evaluar otras conductas, observar si hay diferencias entre grupos, e ir conformando una lista ordenada de acciones proambientales con base en datos cuantitativos que puede ser útil a otros estudios que se interesan en el costo conductual, con el objetivo mayor de entender y promover la CPA (Pieters, 1989), como es la propuesta de Moore y Boldero (2017) quienes consideran que las estrategias de intervención deben plantearse de acuerdo con categorías de CPA basadas en la frecuencia y el costo.

\section{Conclusiones}

Actuar de forma proambiental ya no es solo una alternativa de acción apreciada, sino indispensable en la situación global. Sin embargo, esto no quiere decir que será llevada a cabo incondicionalmente, puesto que no todas las acciones son fáciles o factibles para todas las personas como lo es la elección de transporte en megalópolis, y el acceso a productos cuyo ciclo de vida tenga un menor impacto sobre el entorno. El fomento de la CPA debe tomar en cuenta el costo conductual de su realización. 


\section{Agradecimiento}

Para el Dr José Martínez Guerrero por la facilitación de la información para implementar el método de porcentaje relativo de prioridad.

\section{Declaración de Conflicto de Intereses}

Los autores declaran no tener conflictos de interés de ninguna índole que puedan influir en el desarrollo de la presente investigación. 


\section{Referencias}

Andersson, M., \& von Borgstede, C. (2010). Differentiation of determinants of low-cost and high-cost recycling. Journal of Environmental Psychology, 30, 402-408. https://doi.org/10.1016/j.jenvp.2010.02.003

Bagozzi, P. R., Yi, Y., \& Baumgartner, J. (1990). The level of effort required for behavior as a moderator of the attitude-behavior relation. European Journal of Social Psychology, 20(1), 45-59. https://doi.org/10.1002/ejsp.2420200105

Bamberg, S., \& Möser, G. (2007). Twenty years after Hines, Hungerford, and Tomera: A new meta-analysis of psycho-social determinants of pro-environmental behavior. Journal of Environmental Psychology, 27, 14-25. https://doi.org/10.1016/j. jenvp.2006.12.002

Best, H., \& Kneip, T. (2011). The impact of attitudes and behavioral costs on environmental behavior: A natural experiment on household waste recycling. Social Science Research, 40, 917-930. https://doi.org/10.1016/j.ssresearch.2010.12.001

Byrka, K., Kaiser, F. G., \& Olko, J. (2017). Understanding the acceptance of naturepreservation-related restrictions as the result of the compensatory effects of environmental attitude and behavioral costs. Environment and Behavior, 49, 487508. https://doi.org/10.1177\%2F0013916516653638

Comisión Nacional para el Uso Eficiente de la Energía. (2017). Normas oficiales mexicanas en eficiencia energética en iluminación. En https://www.gob.mx/conuee/accionesy-programas/normas-oficiales-mexicanas-en-eficiencia-energetica-iluminacion

Corral, V. V. (2001). Comportamiento proambiental. Resma

Diekmann, A. \& Preisendörfer, P. (1998). Environmental behavior: discrepancies between aspirations and reality. Rationality and Society, 10(1), 79-102. https://doi.org/10.1 $\underline{177 \% 2 F 104346398010001004}$

Diekmann, A., \& Preisendörfer, P. (2003). Green and greenback: The behavioral effects of environmental attitudes in low-cost and high-cost situations. Rationality and Society, 15(4), 441-472. https://doi.org/10.1177\%2F1043463103154002

Fujii, S. (2006). Environmental concern, attitude toward frugality, and ease of behavior as determinants of pro-environmental behavior intentions. Journal of Environmental Psychology, 26, 262-268. https://doi.org/10.1016/j.jenvp.2006.09.003

Fujii, S., \& Taniguchi, A. (2013). Promoting pro-environmental intentions. Theoretical background and practical applications of travel feedback programs for car use reduction. En V. Corral-Verdugo, C. H. García-Cadena, \& M. Frías-Armenta, M. (Eds), Psychological Approaches to Sustainability (pp. 141-160). Nueva York: Nova Science Publishers.

Gifford, R. (2014). Environmental psychology matters. Annual Review of Psychology, 65, 541-579. https://doi.org/10.1146/annurev-psych-010213-115048 
Kaiser, F. G., \& Byrka, K. (2015). The Campbell paradigm as a conceptual alternative to the expectation of hypocrisy in contemporary attitude research. The Journal of Social Psychology, 155, 12-29. https://doi.org/10.1080/00224545.2014.959884

Kaiser, F. G., Byrka, K. \& Hartig, T. (2010). Reviving Campbell's paradigm for attitude research. Personality and Social Psychology Review, 14, 351-367. https://doi. org/10.1177/1088868310366452

Kaiser, F. G., \& Schultz, P. W. (2009). The attitude-behavior relationship: A test of three models of the moderating role of behavioral difficulty. Journal of Applied Social Psychology, 39, 186-207. https://doi.org/10.1111/j.1559-1816.2008.00435.x

Kollmuss, A., \& Agyeman, J. (2002) Mind the Gap: Why do people act environmentally and what are the barriers to pro-environmental behavior? Environmental Education Research, 8, 239-260. doi: http://dx.doi.org/10.1080/13504620220145401

Martínez-Guerrero, G. J. \& Sánchez-Sosa, S. J. J. (1981). Intervalidación social de estrategias docentes en la Facultad de Psicología. Métodos Docentes, UNAM, 3, $9-48$.

Moore, H. E., \& Boldero, J. (2017). Designing interventions that last: A classification of environmental behaviors in relation to the activities, costs, and effort involved for adoption and maintenance. Frontiers in Psychology, 8, 1-21. https://doi. org/10.3389/fpsyg.2017.01874

Norma nadf-024-ambt-2013. Gaceta oficial del Distrito Federal, 8 de julio de 2015. En: $\quad$ http://data.sedema.cdmx.gob.mx/nadf24/images/infografias/NADF-024AMBT-2013.pdf

Pieters, R. (1989). A note on cost in economic psychology. Journal of Economic Psychology, 10(4), 441-455. https://doi.org/10.1016/0167-4870(89)90037-8

Secretaría de Movilidad [SEMOVI]. (s.f.). Movilidad ciclista en la Ciudad de México. En https://semovi.cdmx.gob.mx/storage/app/media/Feria\%20Transporte\%20de $\% 20$ Pasajeros/MovilidadCiclista 101220.pdf

Starke, A. D., Willemsen. M. C., \& Snijders, C. C. P. (2020). Beyond "one-size-fitsall" platforms: Applying Campbell's paradigm to test personalized energy advice in the Netherlands. Energy Research \& Social Science, 59, 1-12. https://doi. org/10.1016/j.erss.2019.101311

Steg, L., \& Vlek, C. (2009). Encouraring pro-environmental behavior: An integrative review and research agenda. Journal of Environmental Psychology, 29, 309-317. https://doi.org/10.1016/j.jenvp.2008.10.004

Venegas, E. (25 de marzo 2019). 7 de cada 10 mexicanos opta por productos ecológicos. La razón. En línea https://www.razon.com.mx/negocios/7-de-cada-10-mexicanosopta-por-productos-ecologicos/ 